\title{
Time-Domain Joint Parameter Estimation of Chirp Signal Based on SVR
}

\author{
Xueqian Liu and Hongyi Yu \\ Zhengzhou Information Science and Technology Institute, Zhengzhou 450002, China \\ Correspondence should be addressed to Xueqian Liu; liuxqpaper@tom.com
}

Received 12 July 2013; Revised 7 August 2013; Accepted 29 August 2013

Academic Editor: Zhiqiang Ma

Copyright (C) 2013 X. Liu and H. Yu. This is an open access article distributed under the Creative Commons Attribution License, which permits unrestricted use, distribution, and reproduction in any medium, provided the original work is properly cited.

Parameter estimation of chirp signal, such as instantaneous frequency (IF), instantaneous frequency rate (IFR), and initial phase (IP), arises in many applications of signal processing. During the phase-based parameter estimation, a phase unwrapping process is needed to recover the phase information correctly and impact the estimation performance remarkably. Therefore, we introduce support vector regression (SVR) to predict the variation trend of instantaneous phase and unwrap phases efficiently. Even though with that being the case, errors still exist in phase unwrapping process because of its ambiguous phase characteristic. Furthermore, we propose an SVR-based joint estimation algorithm and make it immune to these error phases by means of setting the SVR's parameters properly. Our results show that, compared with the other three algorithms of chirp signal, not only does the proposed one maintain quality capabilities at low frequencies, but also improves accuracy at high frequencies and decreases the impact with the initial phase.

\section{Introduction}

Chirp signals, that is, second-order polynomial phase signals, are common in various areas of science and engineering. For example, in a synthetic aperture radar (SAR) system, when the target is moving, the regulated signals will change into chirp ones after being reflected [1]. The SAR imaging quality may be degraded by shifting and/or defocusing due to inaccurate estimation of instantaneous frequency (IF) and instantaneous frequency rate (IFR), respectively [2]. In optical communications, coding or instability of the laser diode results in chirp phenomenon [3]. The research on estimating these chirp parameters is divided into two parts: one is based on cubic phase function $(\mathrm{CPF})$, even high-order phase function (HPF) [4-6] and the other is based on maximum likelihood (ML) [7-9]. The former has the advantage in fast calculation of IFR, but costs more to find IF. The latter tries its best to maximize an ML function mainly in frequency domain, which involves a twodimensional nonlinear optimization. Unfortunately, there is no exact, closed-form solution for solving this general nonlinear programming problem. The solution either resorts to a burdensome numerical search or is approximated by linearized techniques. Djuric and Kay proposed an efficient time-domain ML estimator [10], which can achieve optimum estimation at a moderate complexity. By extending the phase noise model of [11] to chirp signals, Li et al. derived an improved ML estimator and analyzed its performance in the time domain $[12,13]$. However, both of them are suitable only at high signal-to-noise ratio (SNR), for the reason of approximations of noise phase model and imperfections in phase unwrapping process.

By introducing structural risk minimization (SRM) principle, support vector regression (SVR) exhibits excellent capabilities for generalizing and learning. From the viewpoint of the quadratic relationship between absolute signal phase and time series, therefore, this paper employs SVR to unwrap phases and estimate IF and IFR recursively. We avoid taking the rationality of phase noise model into account by not making any approximations in it. At one time, we reduce the estimation performance's dependence on phase unwrapping process with a proper choice of SVR's parameters. It has been shown that, except for the property of low sensitivity to initial phase and closely approaching the Cramer-Rao lower bound (CRLB) at low frequencies, the proposed algorithm improves its estimation performance at high frequencies. 


\section{The Proposed Algorithm}

2.1. Signal Model. The signal model used here is similar to that in $[10,12]$. The complex, baseband chirp signal polluted by noise is modelled as

$$
\begin{array}{r}
r_{n}=a_{n} A \exp \left\{j\left[\phi+2 \pi f_{d} n T_{s}+2 \pi f_{r}\left(n T_{s}\right)^{2}\right]\right\}+w_{n} \\
n=0, \ldots, N-1 .
\end{array}
$$

Here, $a_{n}$ is an independent symbol; $A>0, f_{d}, f_{r} \in$ $[-0.5,0.5)$, and $\phi \in[-\pi, \pi)$ are the amplitude, deterministic but unknown IF, IFR, and initial phase, respectively; $T_{s}$ is the sampling period; $N$ is the sample size; $w_{n}$ is an independent complex additive white Gaussian noise (AWGN) with zero mean and variance $\sigma^{2}$. For the sake of simplicity, we set $T_{s}=1$ and rearrange (1):

$$
\begin{aligned}
& r_{n}= \exp \left[j\left(\phi+2 \pi f_{d} n+2 \pi f_{r} n^{2}\right)\right] \\
& \times\left\{A+a_{n}^{*} \exp \left[-j\left(\phi+2 \pi f_{d} n+2 \pi f_{r} n^{2}\right)\right] w_{n}\right\} \\
&= \exp \left[j\left(\phi+2 \pi f_{d} n+2 \pi f_{r} n^{2}\right)\right]\left(A+w_{n}^{\prime}\right), \\
& n=0, \ldots, N-1,
\end{aligned}
$$

where $w_{n}^{\prime}=a_{n}^{*} \exp \left[-j\left(\phi+2 \pi f_{d} n+2 \pi f_{r} n^{2}\right)\right] w_{n}$ is still an independent complex AWGN with zero mean and variance $\sigma^{2}$. Absolute phase of $r_{n}$ is presented as

$$
\angle r_{n}=\phi+2 \pi f_{d} n+2 \pi f_{r} n^{2}+\angle\left(A+w_{n}^{\prime}\right), \quad n=0, \ldots, N-1 \text {. }
$$

When SNR $=A^{2} / \sigma^{2}$ is large enough, the approximate model in [10] is expressed as

$$
\begin{aligned}
\angle\left(A+w_{n}^{\prime}\right) & \approx \arctan \frac{\operatorname{Im}\left(w_{n}^{\prime}\right)}{A+\operatorname{Re}\left(w_{n}^{\prime}\right)} \\
& \approx \arctan \frac{\operatorname{Im}\left(w_{n}^{\prime}\right)}{A} \\
& \approx \frac{\operatorname{Im}\left(w_{n}^{\prime}\right)}{A}, \quad n=0, \ldots, N-1 .
\end{aligned}
$$

A more reasonable model proposed in [12] is given:

$$
\begin{aligned}
\angle\left(A+w_{n}^{\prime}\right) & \approx \arcsin \frac{\operatorname{Im}\left(w_{n}^{\prime}\right)}{\left|r_{n}\right|} \\
& \approx \frac{\operatorname{Im}\left(w_{n}^{\prime}\right)}{\left|r_{n}\right|}, \quad n=0, \ldots, N-1 .
\end{aligned}
$$

2.2. SVR-Based Phase Unwrapping Process and Frequency Estimation Algorithm. In (5), there are two approximations still existing. Based on the quadratic relation in (3), this paper utilizes SVR's excellent capability for learning unknown models to unwrap phases and estimate frequency. We yield a training set $S_{k}=\left\{\left(x_{i}^{k}, y_{i}^{k}\right) \mid i=1, \ldots, k\right\}, x_{i}^{k}=i-1, y_{i}^{k}=$ $\angle \widehat{r}_{i-1}$ at time point $k(1 \leq k \leq N)$, where $\angle \widehat{r}_{i-1}$ denotes the estimation value of $\angle r_{i-1}$.

At first, we present a line $f_{k}(x)=\left(\mathbf{w}_{k} \cdot \phi(x)\right)+b_{k}$, where $(\cdot)$ is an inner product operator and $\phi(\cdot)$ is a nonlinear mapping from low to high dimension feature space. And also, we define its $\varepsilon$-insensitive loss function as

$$
\begin{aligned}
L\left(y_{i}^{k}, f_{k}\left(x_{i}^{k}\right)\right) & =\left|y_{i}^{k}-f_{k}\left(x_{i}^{k}\right)\right|_{\varepsilon} \\
& = \begin{cases}0, & \left|y_{i}^{k}-f_{k}\left(x_{i}^{k}\right)\right| \leq \varepsilon_{k} \\
\left|y_{i}^{k}-f_{k}\left(x_{i}^{k}\right)\right|-\varepsilon_{k}, & \text { else, }\end{cases}
\end{aligned}
$$

where $\varepsilon_{k}$ is insensitive loss coefficient at time point $k$.

Next, we assume $f_{k}(x)$ insofar as for $\varepsilon_{k}$ to completely fit all elements of $S_{k}$, and we denote $d_{i}^{k}$ as the distance from point $\left(x_{i}^{k}, y_{i}^{k}\right) \in S_{k}$ to $f_{k}(x)$ :

$$
\begin{aligned}
d_{i}^{k} & =\frac{\left|\left(\mathbf{w}_{k} \cdot \phi\left(x_{i}^{k}\right)\right)+b_{k}-y_{i}^{k}\right|}{\sqrt{1+\left\|\mathbf{w}_{k}\right\|^{2}}} \\
& \leq \frac{\varepsilon_{k}}{\sqrt{1+\left\|\mathbf{w}_{k}\right\|^{2}}}, \quad i=1, \ldots, k .
\end{aligned}
$$

According to (7), we optimize $f_{k}(x)$ through maximizing $\varepsilon_{k} / \sqrt{1+\left\|\mathbf{w}_{k}\right\|^{2}}$, that is, minimizing $\left\|\mathbf{w}_{k}\right\|^{2}$. Thereby, SVR is presented as

$$
\begin{aligned}
& \min J\left(\mathbf{w}_{k}, b_{k}\right)=\frac{1}{2}\left\|\mathbf{w}_{k}\right\|^{2} \\
& \text { s.t. }\left|\left(\mathbf{w}_{k} \cdot \phi\left(x_{i}^{k}\right)\right)+b_{k}-y_{i}^{k}\right| \leq \varepsilon_{k}, \quad i=1, \ldots, k .
\end{aligned}
$$

In fact, fitting errors larger than $\varepsilon_{k}$ always exist. By introducing slack variables $\xi_{i}^{k},\left(\xi^{*}\right)_{i}^{k} \geq 0, i=1, \ldots, k$, and penalty factor $C_{k}$ at time point $k,(8)$ is converted into

$$
\begin{aligned}
& \min J\left(\mathbf{w}_{k}, b_{k}, \boldsymbol{\xi}_{k}, \boldsymbol{\xi}_{k}^{*}\right)=\frac{1}{2}\left\|\mathbf{w}_{k}\right\|^{2}+C_{k} \sum_{i=1}^{k}\left[\xi_{i}^{k}+\left(\xi^{*}\right)_{i}^{k}\right] \\
& \text { s.t. }\left\{\begin{array}{l}
y_{i}^{k}-\left(\mathbf{w}_{k} \cdot \phi\left(x_{i}^{k}\right)\right)-b_{k} \leq \varepsilon_{k}+\xi_{i}^{k} \\
\left(\mathbf{w}_{k} \cdot \phi\left(x_{i}^{k}\right)\right)+b_{k}-y_{i}^{k} \leq \varepsilon_{k}+\left(\xi^{*}\right)_{i}^{k},
\end{array} \quad i=1, \ldots, k,\right.
\end{aligned}
$$

where $\boldsymbol{\xi}_{k}=\left[\xi_{1}^{k}, \xi_{2}^{k}, \ldots, \xi_{k}^{k}\right]^{T}, \boldsymbol{\xi}_{k}^{*}=\left[\left(\xi^{*}\right)_{1}^{k},\left(\xi^{*}\right)_{2}^{k}, \ldots,\left(\xi^{*}\right)_{k}^{k}\right]^{T}$, $[\cdot]^{T}$ is a transpose operator, and $C$ is a positive constant to take compromise in SVR's generalization capability and fitting errors which are denoted by the first and second items of $J\left(\mathbf{w}_{k}, b_{k}\right)$, respectively. 


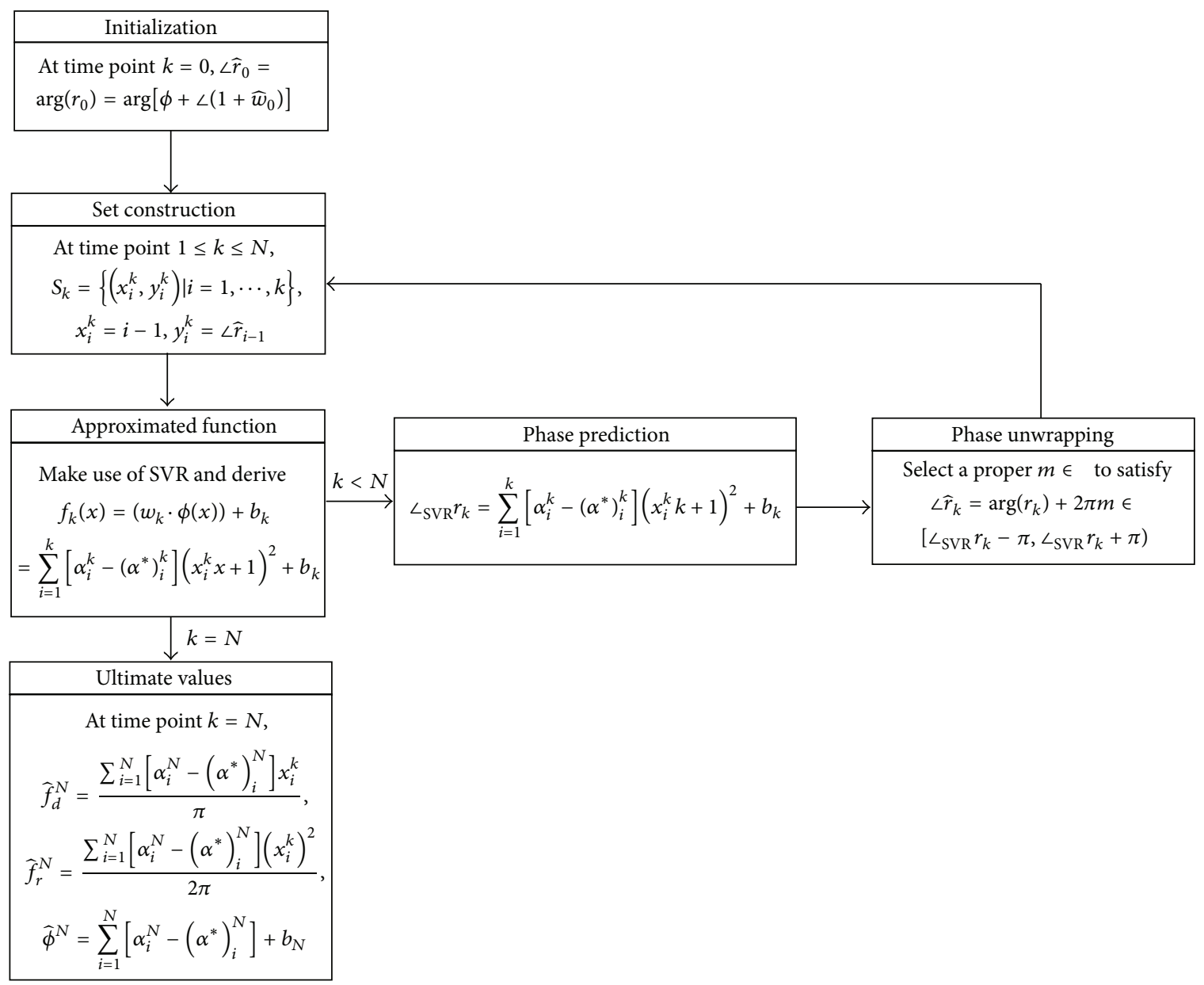

FIGURE 1: Recursive implementation of SVR-based algorithm.

Equation (9) is a strict convex quadratic programming (QP) problem in optimal theories. Then, using Lagrange multiplier method:

$$
\begin{gathered}
\mathbf{w}_{k}=\sum_{i=1}^{k}\left[\alpha_{i}^{k}-\left(\alpha^{*}\right)_{i}^{k}\right] \phi\left(x_{i}^{k}\right), \\
\sum_{i=1}^{k}\left[\left(\alpha^{*}\right)_{i}^{k}-\alpha_{i}^{k}\right]=0, \\
C_{k}-\alpha_{i}^{k}-\gamma_{i}^{k}=0, \quad i=1, \ldots, k, \\
C_{k}-\left(\alpha^{*}\right)_{i}^{k}-\left(\gamma^{*}\right)_{i}^{k}=0, \quad i=1, \ldots, k,
\end{gathered}
$$

where $\alpha_{i}^{k},\left(\alpha^{*}\right)_{i}^{k}, \gamma_{i}^{k}$, and $\left(\gamma^{*}\right)_{i}^{k}$ are the $i$ th Lagrange multipliers of $y_{i}^{k}-\left(\mathbf{w}_{k} \cdot \phi\left(x_{i}^{k}\right)\right)-b_{k} \leq \varepsilon_{k}+\xi_{i}^{k},\left(\mathbf{w}_{k} \cdot \phi\left(x_{i}^{k}\right)\right)+b_{k}-y_{i}^{k} \leq$ $\varepsilon_{k}+\left(\xi^{*}\right)_{i}^{k}, \xi_{i} \geq 0$, and $\left(\xi^{*}\right)_{i}^{k} \geq 0$, respectively.
Substituting (10) into (9), replacing $\left(\phi\left(x_{i}^{k}\right) \cdot \phi\left(x_{j}^{k}\right)\right)$ with quadratic kernel function $K\left(x_{i}, x_{j}\right)=\left(x_{i}^{*} x_{j}+1\right)^{2}$ in this study, and deriving the wolf dual problem of (9):

$$
\begin{aligned}
& \max W\left(\boldsymbol{\alpha}_{k}, \boldsymbol{\alpha}_{k}^{*}\right) \\
& =-\frac{1}{2} \sum_{i, j=1}^{k}\left[\alpha_{i}^{k}-\left(\alpha^{*}\right)_{i}^{k}\right]\left[\alpha_{j}^{k}-\left(\alpha^{*}\right)_{j}^{k}\right]\left(x_{i}^{*} x_{j}+1\right)^{2} \\
& \quad+\sum_{i=1}^{k} y_{i}^{k}\left[\alpha_{i}^{k}-\left(\alpha^{*}\right)_{i}^{k}\right]-\varepsilon_{k} \sum_{i=1}^{k}\left[\alpha_{i}^{k}+\left(\alpha^{*}\right)_{i}^{k}\right] \\
& \text { s.t. } \sum_{i=1}^{k}\left[\alpha_{i}^{k}-\left(\alpha^{*}\right)_{i}^{k}\right]=0 \\
& 0 \leq \alpha_{i}^{k}, \quad\left(\alpha^{*}\right)_{i}^{k} \leq C_{k}, \quad i=1, \ldots, k,
\end{aligned}
$$

where $\boldsymbol{\alpha}_{k}=\left[\alpha_{1}^{k}, \alpha_{2}^{k}, \ldots, \alpha_{k}^{k}\right]^{T}, \boldsymbol{\alpha}_{k}^{*}=\left[\left(\alpha^{*}\right)_{1}^{k},\left(\alpha^{*}\right)_{2}^{k}, \ldots,\left(\alpha^{*}\right)_{k}^{k}\right]^{T}$. 


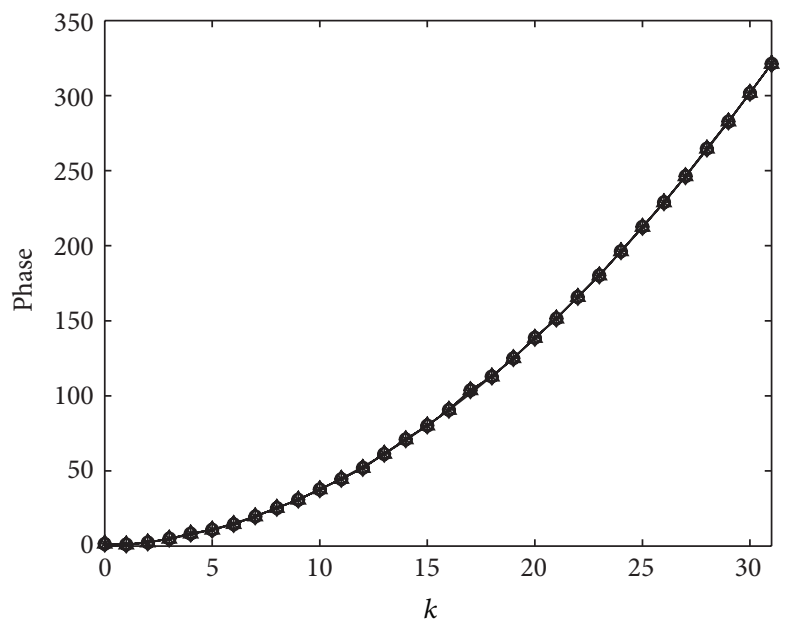

(a)

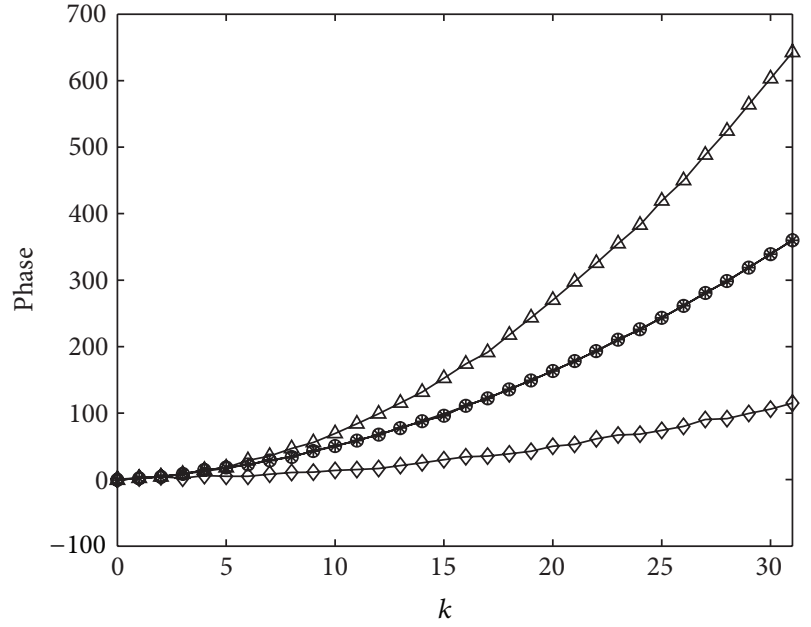

(b)

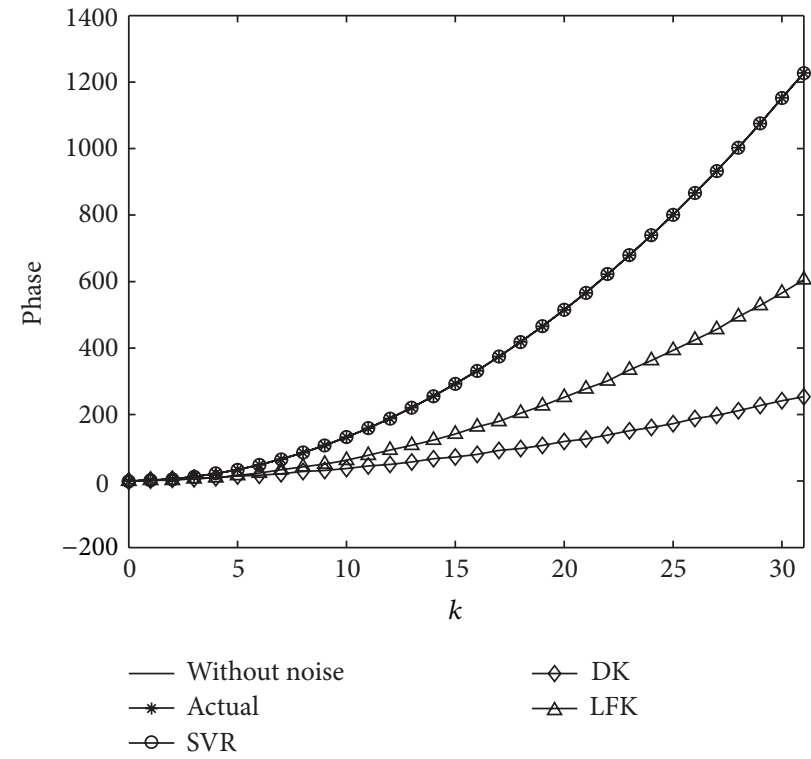

(c)

FIGURE 2: (a) Arbitrary phase unwrapping processes with $f_{d}=0.1, f_{r}=0.05, \phi=0, N=32$, and SNR $=8 \mathrm{~dB}$. (b) Arbitrary phase unwrapping processes with $f_{d}=0.3, f_{r}=0.05, \phi=0, N=32$, and SNR $=8 \mathrm{~dB}$. (c) Arbitrary phase unwrapping processes with $f_{d}=0.1, f_{r}=0.2, \phi=$ $0, N=32$, and $\mathrm{SNR}=8 \mathrm{~dB}$.

We obtain $\boldsymbol{\alpha}_{k}, \boldsymbol{\alpha}_{k}^{*}$ through solving (11). Ultimately, we get the best approximation of (3) by using Karush-Kuhn-Tucker (KKT) conditions:

$$
\begin{aligned}
f_{k}(x) & =\left(\mathbf{w}_{k} \cdot \phi(x)\right)+b_{k} \\
& =\sum_{i=1}^{k}\left[\alpha_{i}^{k}-\left(\alpha^{*}\right)_{i}^{k}\right]\left(x_{i}^{*} x_{j}+1\right)^{2}+b_{k},
\end{aligned}
$$

where $b_{k}=y_{i}^{k}-\sum_{j=1}^{k}\left[\alpha_{j}^{k}-\left(\alpha^{*}\right)_{j}^{k}\right]\left(x_{i}^{*} x_{j}+1\right)^{2}-\varepsilon_{k}, \alpha_{i}^{k} \in\left(0, C_{k}\right)$ or $b_{k}=y_{i}^{k}-\sum_{j=1}^{k}\left[\alpha_{j}^{k}-\left(\alpha^{*}\right)_{j}^{k}\right]\left(x_{i}^{*} x_{j}+1\right)^{2}+\varepsilon_{k},\left(\alpha^{*}\right)_{i}^{k} \in\left(0, C_{k}\right)$ is bias at time point $k$.

Not only does (6) guarantee less fitting errors, but it also possesses a good generalization capability. It means that, after learning previous unwrapped phases $\angle \widehat{r}_{0}, \ldots, \angle \widehat{r}_{k-1},(6)$ can predict the variation trend of a phase efficiently for the next time point $k$ and derive $L_{\mathrm{SVR}} r_{k}=\sum_{i=1}^{k}\left[\alpha_{i}^{k}-\left(\alpha^{*}\right)_{i}^{k}\right]\left(x_{i}^{*} k+1\right)^{2}+$ $b_{k}$. The phase $\angle \widehat{r}_{k}$ is then unwrapped within a $2 \pi$ interval centered around $L_{\mathrm{SVR}} r_{k}$, by adding multiples of $\pm 2 \pi$ to the principal value of $\angle \widehat{r}_{k}$ when the absolute difference between $L_{\text {SVR }} r_{k}$ and the principal value of $\angle \widehat{r}_{k}$ is greater than $\pi$. Namely, we select a proper $m$ to satisfy $\angle \widehat{r}_{k}=\arg \left(r_{k}\right)+2 \pi m \epsilon$ $\left[L_{\mathrm{SVR}} r_{k}-\pi, L_{\mathrm{SVR}} r_{k}+\pi\right)$. Till time point $k=N$, we get $\widehat{f}_{d}^{N}=$ $\left(\sum_{i=1}^{N}\left[\alpha_{i}^{N}-\left(\alpha^{*}\right)_{i}^{N}\right] x_{i}^{N}\right) / \pi, \widehat{f}_{r}^{N}=\left(\sum_{i=1}^{N}\left[\alpha_{i}^{N}-\left(\alpha^{*}\right)_{i}^{N}\right]\left(x_{i}^{N}\right)^{2}\right) / 2 \pi$, and $\widehat{\phi}^{N}=\sum_{i=1}^{N}\left[\alpha_{i}^{N}-\left(\alpha^{*}\right)_{i}^{N}\right]+b_{N}$ as the ultimate estimation values. This algorithm can be implemented recursively in time, as shown schematically in Figure 1. 


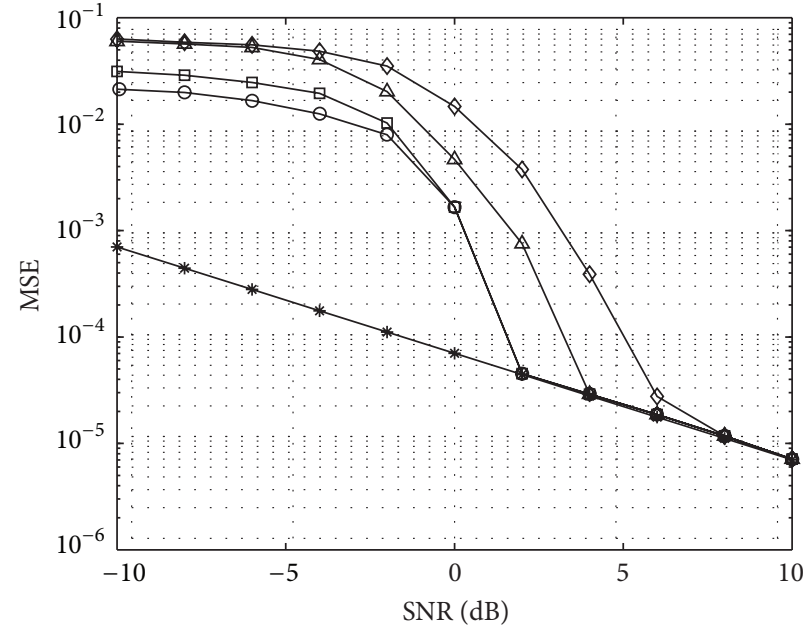

(a)

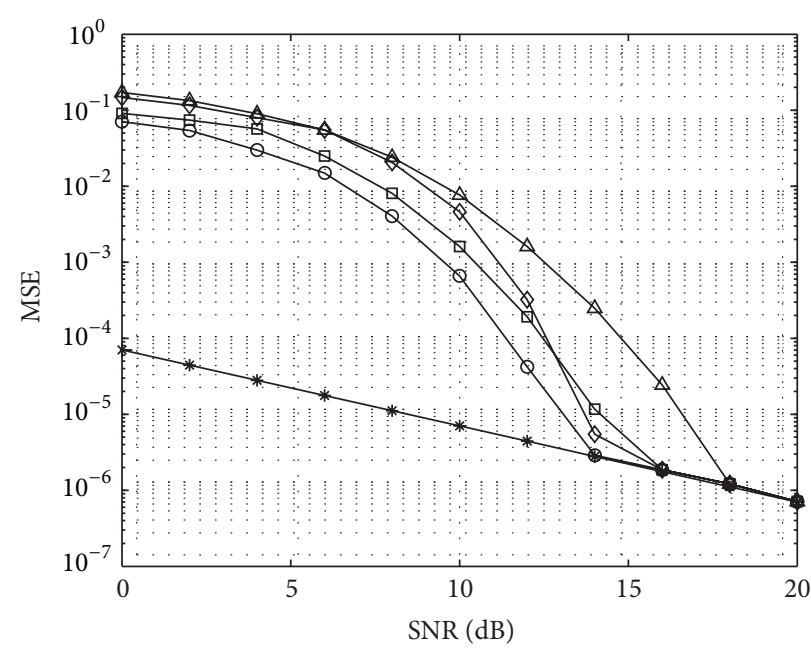

(b)

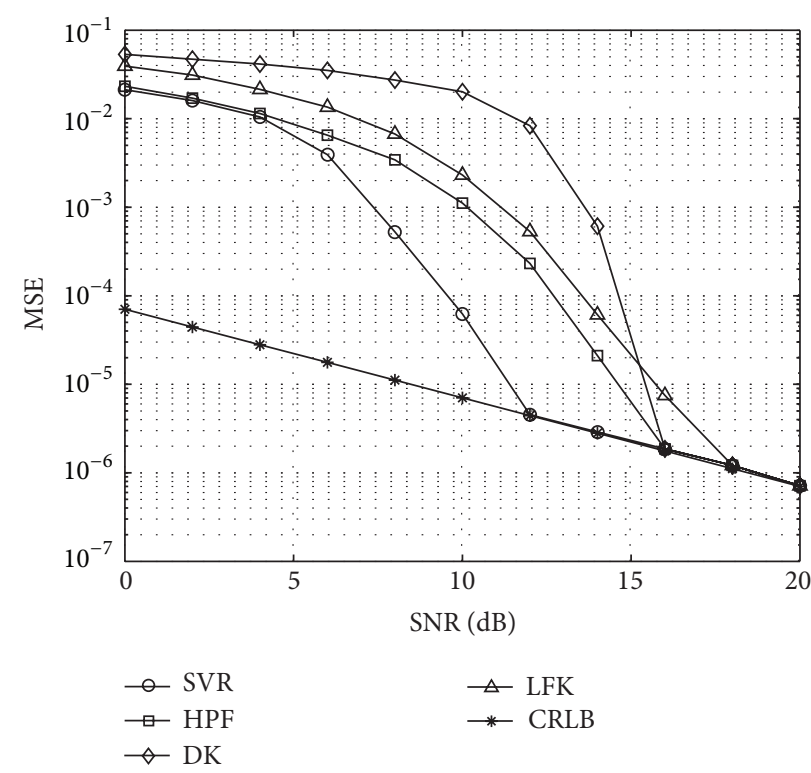

(c)

Figure 3: (a) MSE of IF with $f_{d}=0.1, f_{r}=0.05, \phi=0$, and $N=32$. (b) MSE of IF with $f_{d}=0.3, f_{r}=0.05, \phi=0$, and $N=32$. (c) MSE of IF with $f_{d}=0.1, f_{r}=0.2, \phi=0$, and $N=32$.

2.3. SVR's Parameter Settings. Setting SVR's parameters is a difficult problem, but has a pronounced impact on SVR's performance, for example, insensitive loss coefficient $\varepsilon$, penalty factor $C$. There is no complete theoretical basis or explicit closed form. Cross-validation is a widely used method until now, but it is complex and time consuming. In this study, we formulate proper parameter values by understanding SVR's theory and integrating a large number of references and experiments.

When $k$ is small, imperfect phase unwrapping at a particular time point can easily have an impact on the variation trend of $f(x)$. In order to avoid this discrepancy, a new model must be constructed with the aptitude of better generalization capability. As $k$ increases, its necessity decreases contrarily, which is due to the degrading impact of improperly unwrapped phase. Simple speaking, the model's generalization capability is inversely proportional to the size of the set $S$.

Intuitively, insensitive loss coefficient $\varepsilon$ is the vertical height of $\varepsilon$-tube. The larger $\varepsilon$ is, the less support vectors there are. Nevertheless, too large $\varepsilon$ will cause unfixable $b$. Noted that, if the predicted phase is in the vicinity of $\pi$ 's odd times, the estimation performance is deteriorated rapidly for its ambiguous phase characteristic. Selecting a proper $\varepsilon$ can reduce this impact. At the same time, $\varepsilon$ is inversely proportional to SNR, so insensitive loss coefficient at time point $k(1 \leq k \leq N)$ is given by [14]

$$
\varepsilon_{k}=\tau \sqrt{\frac{1}{\operatorname{SNR}} \frac{\ln k}{k}}, \quad k=1, \ldots, N
$$




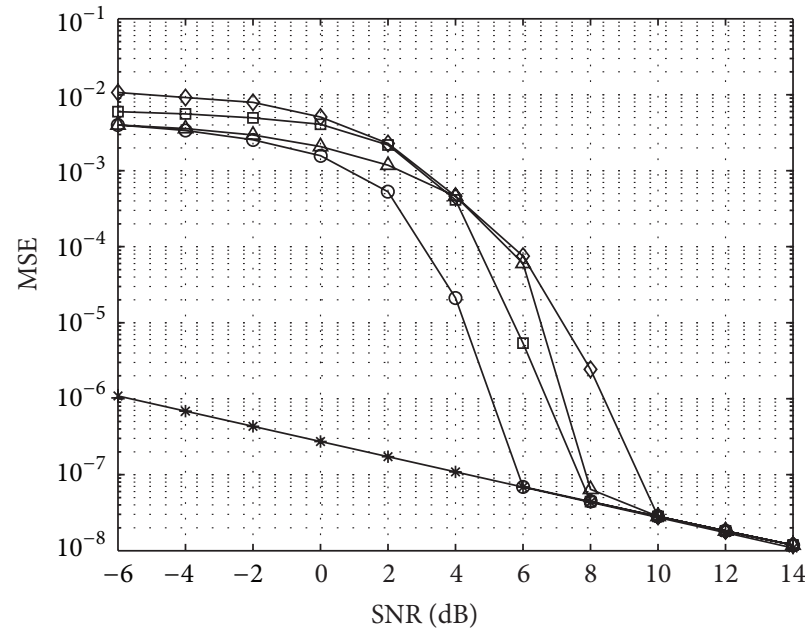

(a)

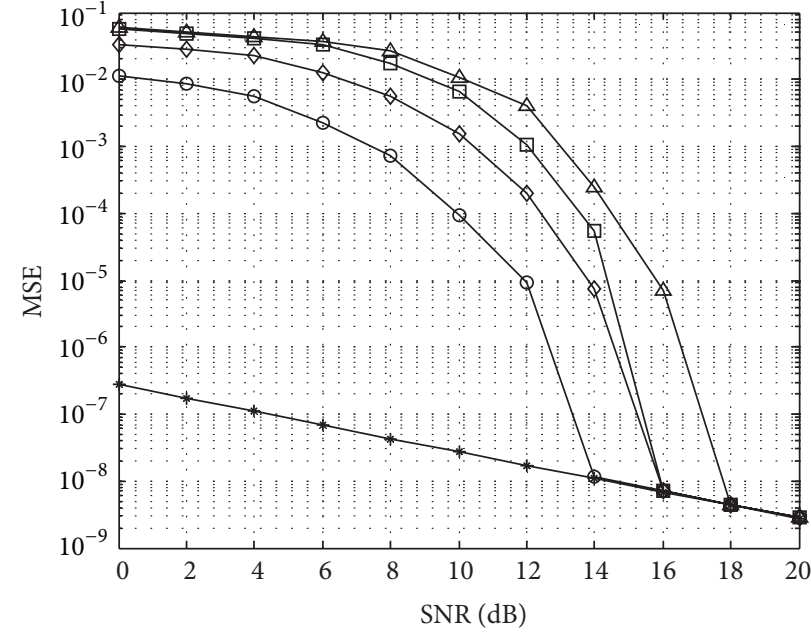

(b)

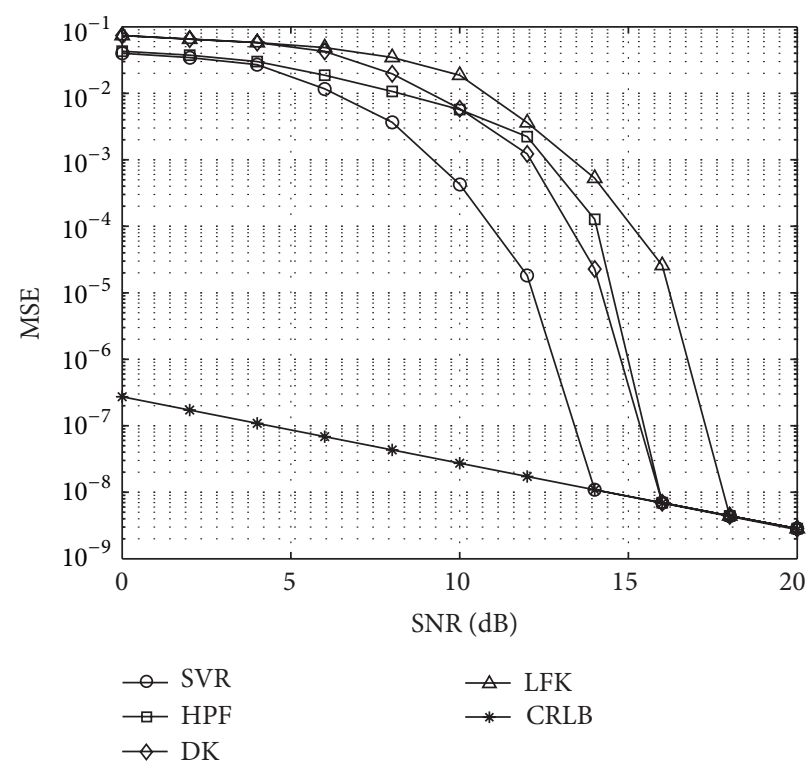

(c)

FIGURE 4: (a) MSE of IFR with $f_{d}=0.1, f_{r}=0.05, \phi=0$, and $N=32$. (b) MSE of IFR with $f_{d}=0.3, f_{r}=0.05, \phi=0$, and $N=32$. (c) MSE of IFR with $f_{d}=0.1, f_{r}=0.2, \phi=0$, and $N=32$.

where $\tau$ is a positive constant, and $\tau=0.8$ is set in this study after experimental comparisons and validations, and SNR is assumed to be known.

Penalty factor $C$ controls the penalty degree of vectors outside the $\varepsilon$-tube and determines SVR's generalization capability. $C$ is directly proportional to the sample size and SNR. As (12) is a line in a two-dimension plane, the first item of target function during solving SVR's QP problem is directly proportional to its slope; so a very small $C$ will result in a horizontal and underfitting $C$. Inspired by $[14,15]$, penalty factor at time point $k(1 \leq k \leq N)$ is given by

$$
\begin{array}{r}
C_{k}=\delta \sqrt{\mathrm{SNR}} \sqrt[3]{k} \max \left(\left|\overline{g_{k}}-\lambda \sigma_{k}\right|,\left|\overline{g_{k}}+\lambda \sigma_{k}\right|\right), \\
k=1, \ldots, N ;
\end{array}
$$

where $\overline{g_{k}}=(1 / k) \sum_{n=0}^{k-1}\left|y_{n}\right|^{2}, \sigma_{k}=\sqrt{(1 / k) \sum_{n=0}^{k-1}\left(\left|y_{n}\right|^{2}-\overline{g_{k}}\right)^{2}}$, $y_{n}=\angle \widehat{r}_{n-1}$ is a section of the element of training set $S_{k}$; as (13), SNR is assumed to be known; $\delta, \lambda$ are positive constants and $\delta=0.1, \lambda=0.5$ are set in this study, by the same way as $\tau$.

\section{Results and Analyses}

We have compared the proposed algorithm entitled as SVR estimator with the other three: the HPF estimator proposed in [6]; the DK estimator proposed in [10]; the LFK estimator proposed in [12].

3.1. Phase Unwrapping Process. Because HPF estimator does not need the phase unwrapping process, Figure 2 illustrates 


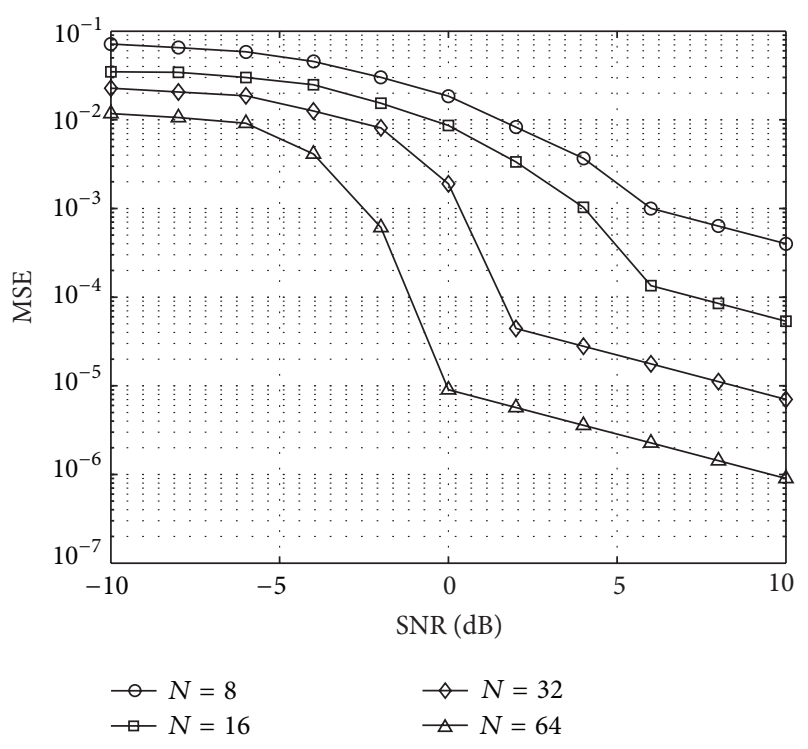

FIGURE 5: MSE of IF of SVR estimator with $f_{d}=0.1, f_{r}=0.05, \phi=$ 0 .

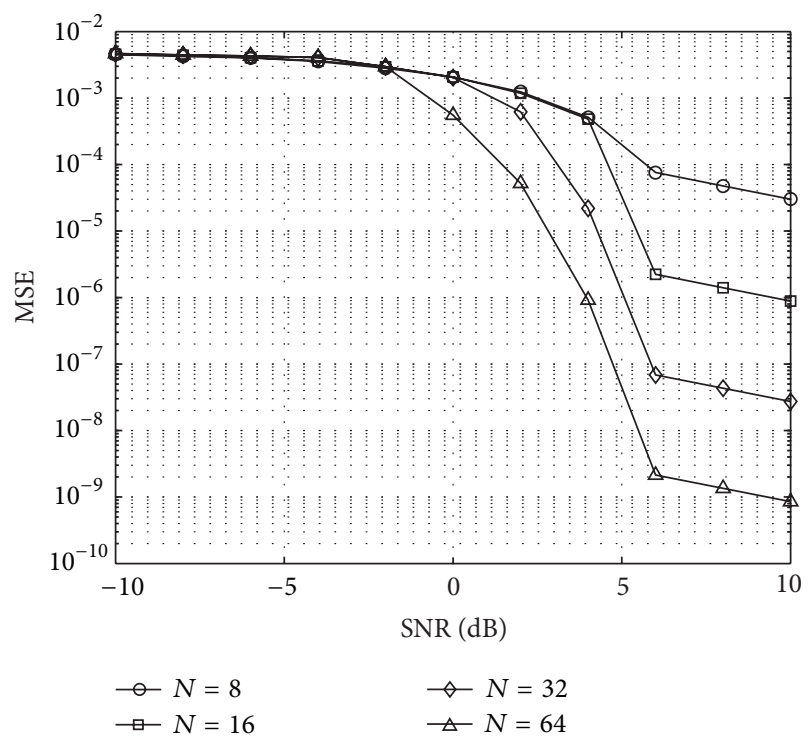

FIGURE 6: MSE of IFR of SVR estimator with $f_{d}=0.1, f_{r}=0.05, \phi=$ 0 .

the arbitrary phase unwrapping processes of the other three, while $f_{d}=0.1, f_{r}=0.05, f_{d}=0.3, f_{r}=0.05$, and $f_{d}=$ $0.1, f_{r}=0.2$. The number of Monte Carlo experiments is 10000 , and $\phi=0, N=32$, and $\mathrm{SNR}=8 \mathrm{~dB}$. It is shown that SVR estimator can unwrap phase accurately, whatever the value of $f_{d}$ and $f_{r}$ is. However, errors emerge in DK and LFK ones as $f_{d}$ and $f_{r}$ increase.

3.2. Estimation Performance. Figures 2, 3, and 4 illustrate the MSE curves of IF and IFR versus SNR, respectively, where MSEs are defined as $E\left[\left(\widehat{f}_{d}-f_{d}\right)^{2}\right], E\left[\left(\hat{f}_{r}-f_{r}\right)^{2}\right], \widehat{f}_{d}, \widehat{f}_{r}$ are the estimation values of $f_{d}, f_{r}$. CRLBs are given by [12]

$$
\begin{aligned}
\mathrm{CRLB}_{f_{d}}= & \frac{1}{\operatorname{SNR}} \frac{6(8 N-11)(2 N-1)}{(2 \pi)^{2} N(N-1)\left(N^{3}+N^{2}-4 N-4\right)}, \\
\mathrm{CRLB}_{f_{r}}= & \frac{1}{\operatorname{SNR}} \frac{360}{(2 \pi)^{2} N(N-1)\left(N^{3}+N^{2}-4 N-4\right)}, \\
& \mathrm{CRLB}_{\phi}=\frac{1}{\operatorname{SNR}} \frac{3\left(3 N^{2}-3 N+2\right)}{2 N\left(N^{2}+3 N+2\right)} .
\end{aligned}
$$

It is shown that whether $f_{d}=0.1, f_{r}=0.05, f_{d}=$ $0.3, f_{r}=0.05$, or $f_{d}=0.1, f_{r}=0.2$, SVR estimator is the best one during both IF and IFR estimations all the while; also, MSE performances of IF and IFR totally decrease as $f_{d}$ and $f_{r}$ increase, but SVR estimator is the most robust and has had a great advantage already when $f_{d}=0.3, f_{r}=0.05$ or $f_{d}=0.1, f_{r}=0.2$.

3.3. Impact of the Sample Size N. Everything is as in Figure 2 other than $f_{d}=0.1, f_{r}=0.05$; the MSE curves of IF and IFR of SVR estimator versus SNR while $N$ is $8,16,32$, and 64 are plotted in Figures 5 and 6, respectively. It is clear that as $N$ increases, MSE performances of IF and IFR of SVR estimator are both improved.

3.4. Impact of Initial Phase $\phi$. Everything is as in Figure 3 except that $\phi=0.4 \pi, 0.8 \pi$; the MSE curves of IF and IFR are plotted against SNR in Figures 7 and 8 , respectively. Comparing with Figures 3(a) and 4(a), we can see that SVR estimator is immune to $\phi$, but the other three are not.

3.5. Computational Complexity. Because we translate SVR into QP problem and need to search the minimums during the process, we can not derive the explicit form of the computational complexity of SVR estimator. So everything is as in Figure 2 except that the number of Monte Carlo experiments is $100, \mathrm{SNR}$ is $8 \mathrm{~dB}$, and $f_{d}=0.1, f_{r}=0.05$; the consuming times are listed in Table 1 , while $N$ is $8,16,32$, and 64, respectively. The running computer is ASUS-PC1111 having, Intel(R) Pentium 2.13 GHz CPU and 2.00 GB RAM.

We can see that SVR estimator's consuming times are more than the others', especially when $N$ becomes larger. As a matter of fact, however, it is acceptable and tolerable.

\section{Conclusions}

Phase unwrapping process is a key point in phase-based frequency estimation of chirp signal. Firstly, we adopt SVR to learn the unwrapped phases at previous time points, predict the variation trend of phase efficiently, and derive the estimation value for the next time point. Once acquired, in terms of relationship between absolute signal phase and time series, we address a simple and effective frequency estimation algorithm of chirp signal. The proposed algorithm completely exhibits its advantages of higher estimation accuracy, lower sensitivity of frequency, and initial phase, by sacrificing more consuming times.

Because SVR predicts the curve's variation trend merely in terms of training set which consists of previous points' 


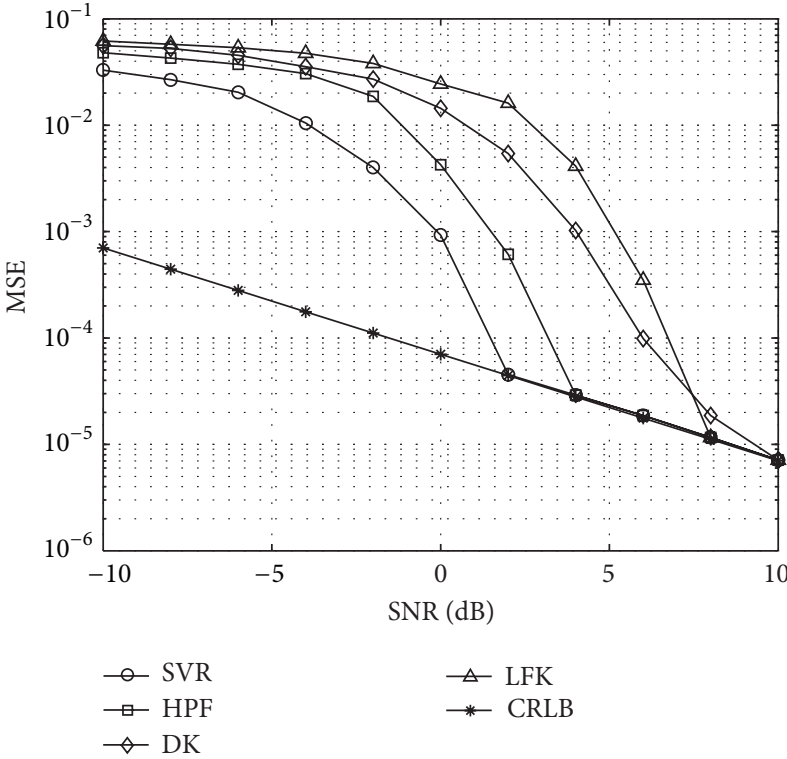

(a)

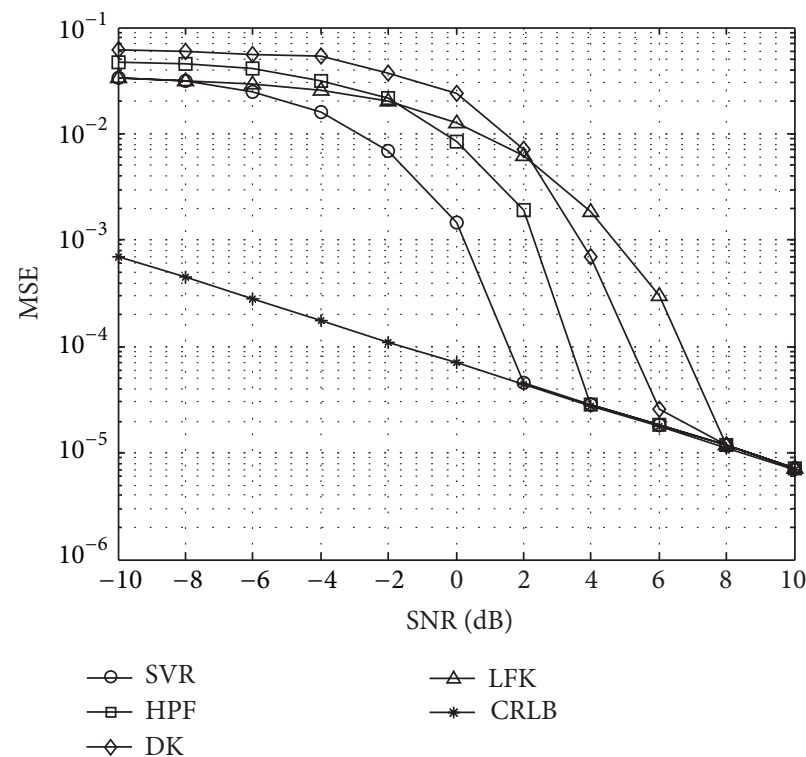

(b)

FIGURE 7: (a) MSE of IF with $f_{d}=0.1, f_{r}=0.05, \phi=0.4 \pi$, and $N=32$. (b) MSE of IF with $f_{d}=0.1, f_{r}=0.05, \phi=0.8 \pi$, and $N=32$.

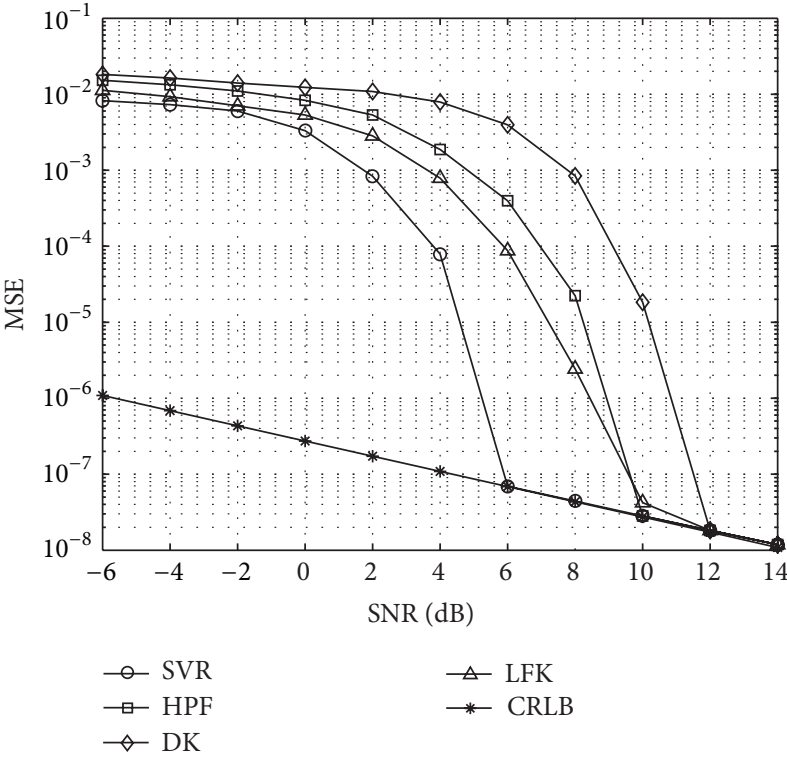

(a)

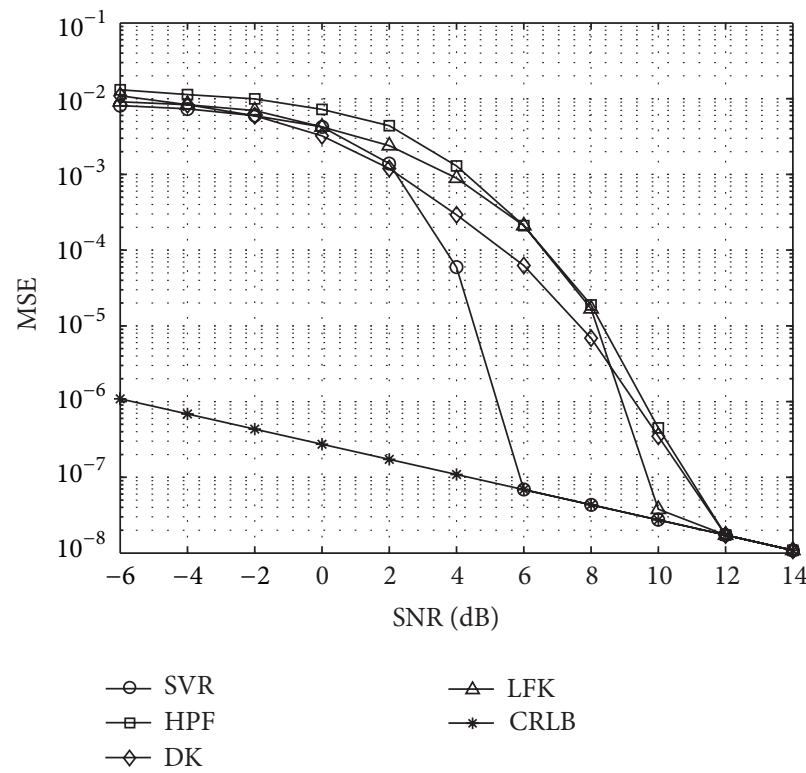

(b)

Figure 8: (a) MSE of IFR with $f_{d}=0.1, f_{r}=0.05, \phi=0.4 \pi$, and $N=32$. (b) MSE of IFR with $f_{d}=0.1, f_{r}=0.05, \phi=0.8 \pi$, and $N=32$.

TABLE 1: Consuming time with different $N(\mathrm{~ms})$.

\begin{tabular}{lcccc}
\hline Algorithm & $N=8$ & $N=16$ & $N=32$ & $N=64$ \\
\hline HPF & 380 & 942 & 3721 & 10654 \\
DK & 203 & 476 & 1098 & 3711 \\
LFK & 299 & 784 & 2362 & 8643 \\
SVR & 437 & 1107 & 4805 & 13074 \\
\hline
\end{tabular}

values, we even can estimate the frequency of chirp signal under the nonGaussian condition by the same way.
Stressing that the proposed algorithm learns training set and gets the approximate values of SVR's insensitive loss coefficient $\varepsilon$ and penalty factor $C$, as a next step, therefore, improving SVR's parameter setting is an important research point.

\section{Acknowledgment}

This research is supported by the National Natural Science Foundation of China (Grant no. 61201380). 


\section{References}

[1] J. C. Curlander and R. N. McDonough, Synthetic Aperture Radar Systems and Signal Processing, Wiley, New York, NY, USA, 1992.

[2] J. Xu, Y. Peng, and X. Xia, "Parametric autofocus of SAR imaging-inherent accuracy limitations and realization," IEEE Transactions on Geoscience and Remote Sensing, vol. 42, no. 11, pp. 2397-2411, 2004.

[3] R. Ramaswami, K. N. Sivarajan, and G. H. Sasaki, Optical Networks: A Practical Perspective, Morgan Kaufmann, San Mateo, Calif, USA, 1998.

[4] P. O'Shea, "A new technique for instantaneous frequency rate estimation," IEEE Signal Processing Letters, vol. 9, no. 8, pp. 251252, 2002.

[5] P. Wang, I. Djurović, and J. Yang, "Generalized high-order phase function for parameter estimation of polynomial phase signal," IEEE Transactions on Signal Processing, vol. 56, no. 7, pp. 30233028, 2008.

[6] P. Wang, H. Li, I. Djurović, and B. Himed, "Performance of instantaneous frequency rate estimation using high-order phase function," IEEE Transactions on Signal Processing, vol. 58, no. 4, pp. 2415-2421, 2010.

[7] M. Benidir and A. Ouldali, "Polynomial phase signal analysis based on the polynomial derivatives decompositions," IEEE Transactions on Signal Processing, vol. 47, no. 7, pp. 1954-1965, 1999.

[8] P. O'Shea, "A fast algorithm for estimating the parameters of a quadratic FM signal," IEEE Transactions on Signal Processing, vol. 52, no. 2, pp. 385-393, 2004.

[9] S. Ma, J. Jiang, and Q. Meng, "A fast, accurate and robust method for joint estimation of frequency and frequency rate," in Proceedings of the 2011 International Symposium on Intelligent Signal Processing and Communications Systems (ISPACS '11), pp. 1-6, Chiang Mai, Thailand, December 2011.

[10] P. M. Djuric and S. M. Kay, "Parameter estimation of chirp signals," IEEE Transactions on Acoustics, Speech, and Signal Processing, vol. 38, no. 12, pp. 2118-2126, 1990.

[11] H. Fu and P.-Y. Kam, "Phase-based, time-domain estimation of the frequency and phase of a single sinusoid in AWGN-the role and applications of the additive observation phase noise model," IEEE Transactions on Information Theory, vol. 59, no. 5, pp. 3175-3188, 2013.

[12] Y. Li, H. Fu, and P. Y. Kam, "Improved, approximate, timedomain ML estimators of chirp signal parameters and their performance analysis," IEEE Transactions on Signal Processing, vol. 57, no. 4, pp. 1260-1272, 2009.

[13] Y. Li and P. Y. Kam, "Improved chirp parameter estimation using signal recovery method, approximate, time-domain ML estimators of signal $s$ and their performance analysis," in Proceedings of the IEEE 71st Vehicular Technology Conference, pp. 1-5, Taipei, Taiwan, May 2010.

[14] V. Cherkassky and Y. Ma, "Selection of meta-parameters for support vector regression," in Proceedings of the International Conference on Artificial Neural Networks, pp. 687-693, Madrid, Spain, August 2002.

[15] V. Cherkassky, X. Shao, F. M. Mulier, and V. N. Vapnik, "Model complexity control for regression using VC generalization bounds," IEEE Transactions on Neural Networks, vol. 10, no. 5, pp. 1075-1089, 1999. 


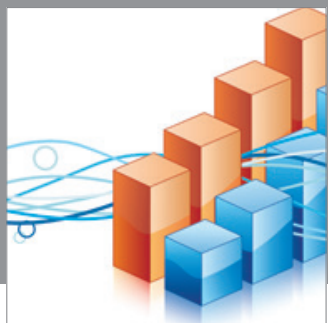

Advances in

Operations Research

mansans

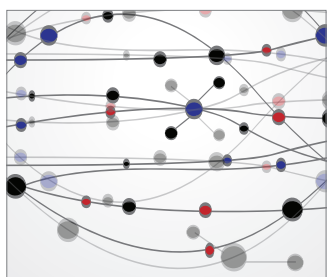

The Scientific World Journal
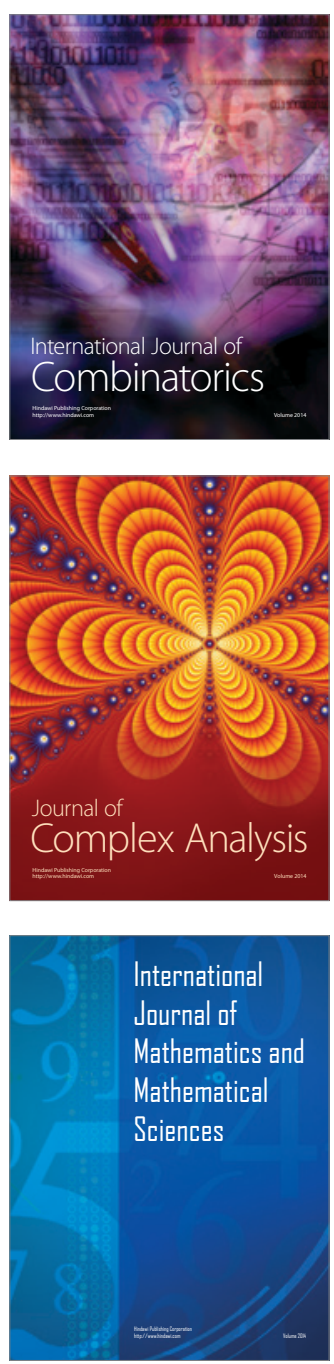
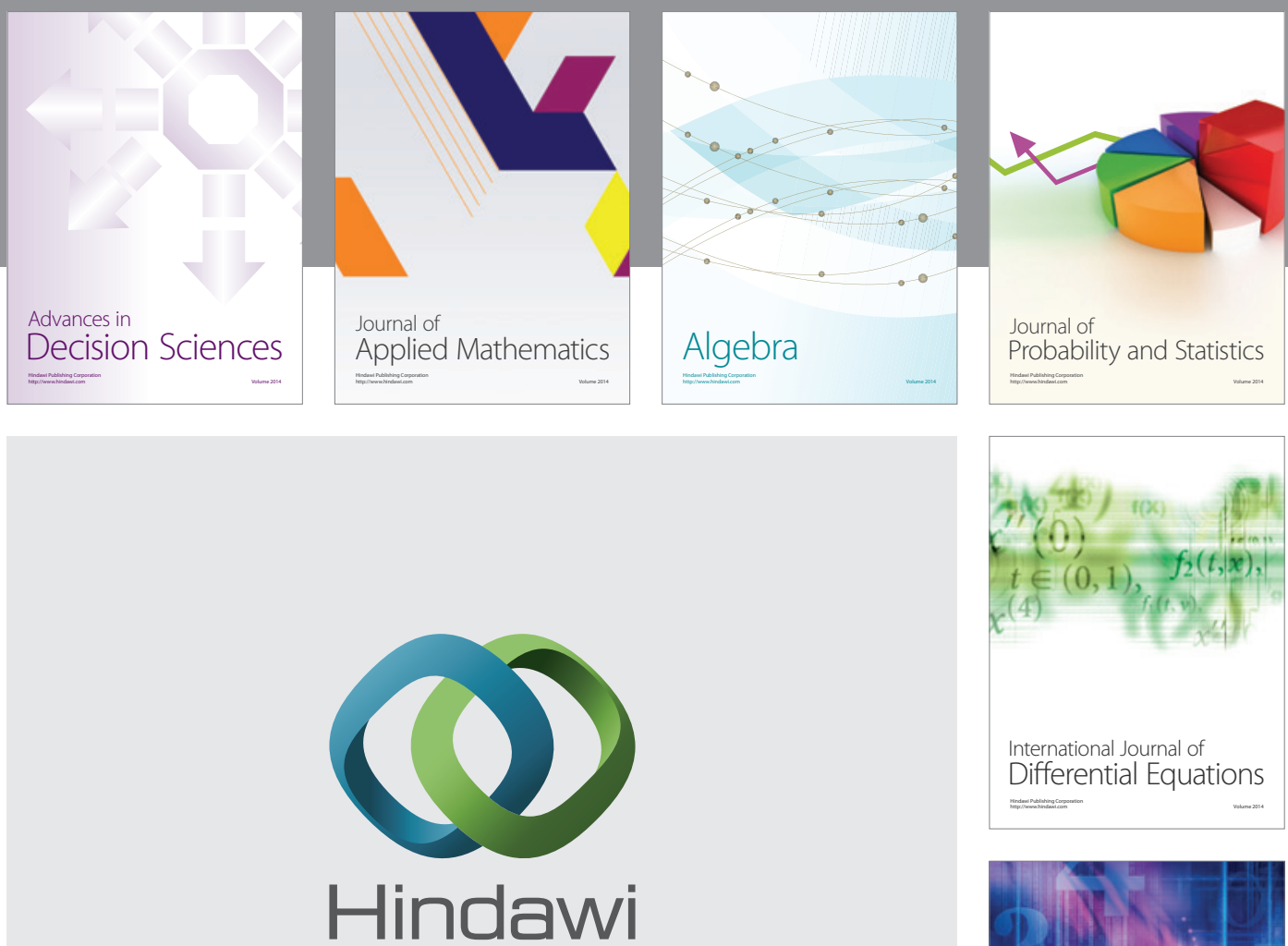

Submit your manuscripts at http://www.hindawi.com
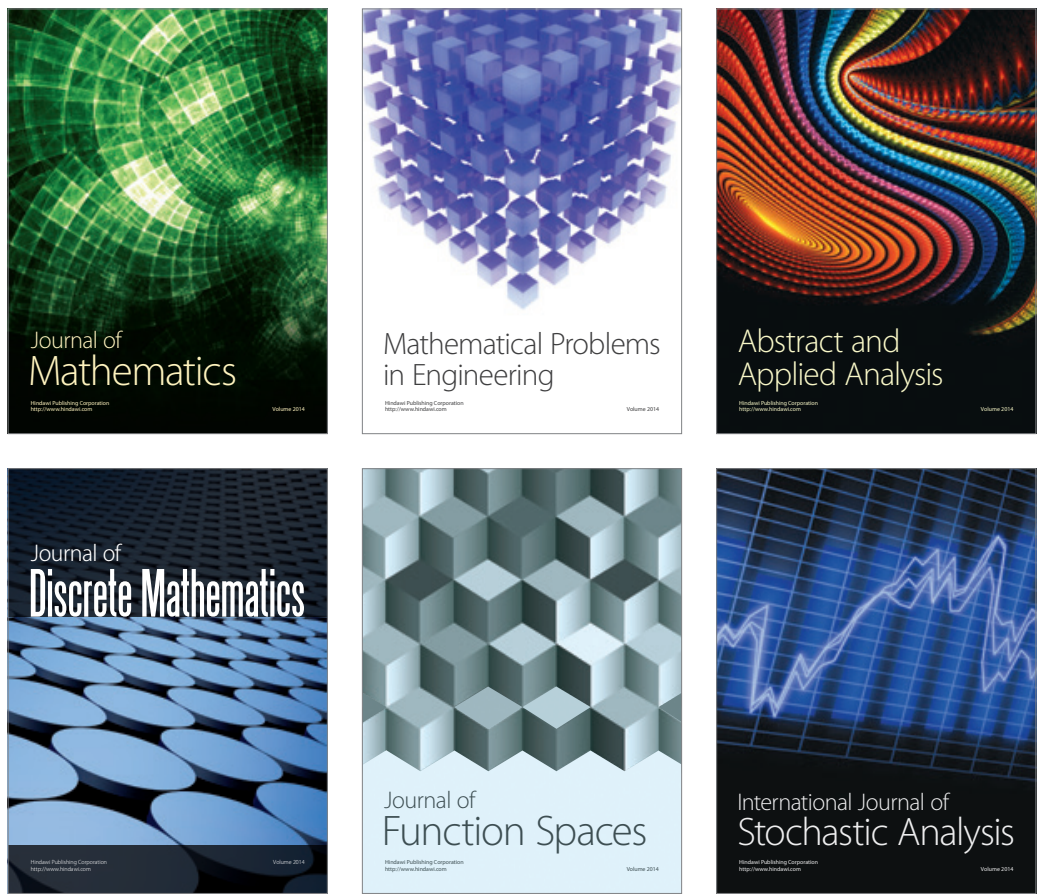

Journal of

Function Spaces

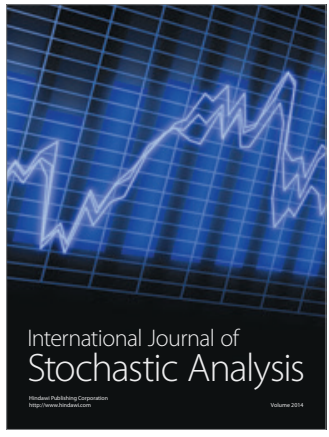

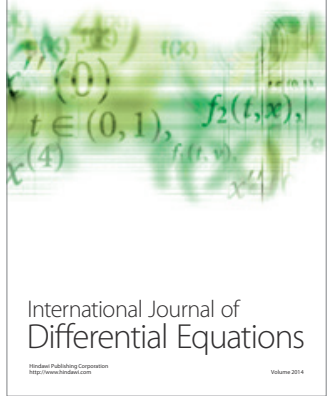
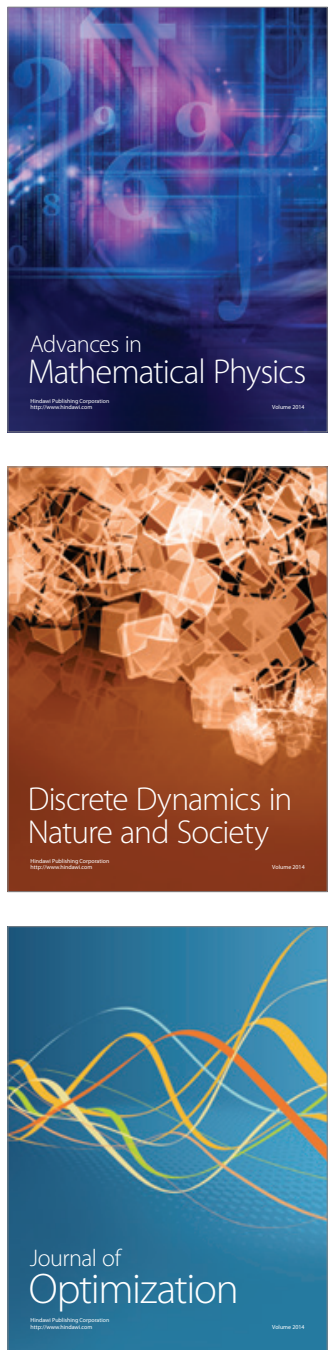